\title{
Pre-Analysis Plan \\ Take Up and Impact of Digital Repayment in Microfinance
}

\author{
Isabelle Cohen and Denise Ferris \\ January 10, 2022
}

\section{Introduction}

This study uses a randomized experiment to evaluate the effects of introducing mobile money-based repayments for microfinance borrowers in Uganda. A baseline survey was conducted in September and October 2021 for a randomly selected subsample of 2,100 borrowers. Randomization was conducted on November 17, 2021 for all 10,283 borrowers, and the rollout is scheduled for January 17, 2022. The endline is planned for July 2022, with the date to be finalized as the study progresses.

Study participants will be randomized at the group level into the following conditions:

- Control: Loan repayment will be done via cash

- Mobile Money Treatment: All borrowers will be asked to repay via mobile money

- Individual Choice Treatment: Borrowers will be able to repay via either cash or mobile money (based on a pre-randomization choice)

The experiment will test the behavioral and social effects of switching to mobile money-based repayment for microfinance loans. By comparing the three groups, it will be possible to assess the effects on personal and business outcomes (using survey data), as well as on loan repayment performance and group meeting attendance using BRAC administrative data.

Note that although the study also includes a baseline experiment designed to test information provision around mobile money use and safety, this sub-experiment was conducted prior to the finalization of the pre-analysis plan (PAP), and therefore is not included here. Any analysis done utilizing this sub-experiment will be identified accordingly. This PAP instead focuses on the main interventions of the experiment; although the information treatment is mentioned briefly herein, it is only discussed analytically so far as it pertains to interactions with the treatment effect of interest.

\section{Overview of the Study}

\subsection{Location, Partner and Population}

This study will be conducted in partnership with BRAC Uganda Bank Limited (BUBL), one of the largest providers of financial services to the poor in Uganda. They offer financial services to micro, small and medium entrepreneurs across the country, and serve primarily women. 
This study will take place across nine BUBL branches and satellites, located in a mix of urban, peri-urban and rural environments. Specifically, these branches are: Iganga Nkono, Kabuusu, Kalerwe, Masaka, Mbarara, Mityana, Mukono Central and Semuto.

The subject population consists of female microfinance clients, the majority of whom operate small businesses, and all of whom have access to a mobile money and BUBL bank account (a requirement for borrowing). The women are part of BUBL microfinance groups of up to 30 women and make payments on their loans at weekly group meetings. Although loans are dispersed in cash on an individual liability basis (with an external guarantor), repayment is done in a weekly group meeting as with other microfinance providers worldwide.

\subsection{Main Intervention}

The main intervention in this project is the introduction of mobile money-based repayment for microfinance loans, with randomized assignment between treatment and control at the group level. Mobile money-based repayment will be implemented within a group in one of two ways, corresponding to the two treatment arms of the study.

Mobile Money Treatment: All borrowers in this arm will be asked to repay via mobile money. Borrowers will be allowed to transact digitally between their mobile money wallet and their bank account.

Individual Choice Treatment: All borrowers in this arm will be allowed to repay either via cash or mobile money, based on a choice made prior to randomization. Borrowers who selected mobile money will be allowed to transact digitally between their mobile money wallet and their bank account.

Control: We will also have a control group in which borrowers repay with cash at the group meeting, as they did prior to the implementation of the study.

In practice, borrowers will pay by "pushing" money from their mobile money wallet to the BUBL bank account, from which it will be debited. These accounts also function as savings accounts. In practice, if an individual with a savings balance has a positive balance on the due date of the loan, that balance will be automatically debited towards the repayment of the loan. Individuals can also make payments using money stored in their mobile money wallet, or by making a cash payment to a mobile money agent (who are readily available for the entire sample, generally within a fifteen-to-thirty-minute walk).

Payments can be made using mobile money accounts from either of the two main cellular carriers in Uganda, Airtel and MTN. Both Airtel and MTN impose transaction fees, including fees for sending money from one's mobile money wallet to one's bank account, pulling money from one's bank account to one's mobile money wallet, or withdrawing money from one's mobile money wallet into cash. Although these fees scale proportionately with transactions, they do so decreasingly, meaning the percentage of the transaction paid is lower as the transaction 
increases across 12 different bands for sending money, and 9 different bands for receiving money. Someone making a transfer of 50,000 UGX, like the average payment due for a microfinance loan, would expect to pay 1,500 UGX making that transaction via MTN and 2,000 making that transaction via Airtel, paying $3 \%$ or $4 \%$ of the transfer amount in fees, respectively. For this study, the cost of these fees will be paid by the borrowers.

It is worth noting that despite the switch in payment modality in several of the treatments, borrowers will still be asked to attend once-weekly group meetings. For the control group and those repaying with cash in the individual choice treatment, repayments will be made at this time; for those in the mobile money or repaying with mobile money in the individual choice treatment, loans installments are still due on the date of the group meeting, even if the repayment is made via mobile money. It is worth nothing that while attendance is strongly encouraged, it is not incentivized (financially or otherwise).

In practice, it is unlikely that BUBL will force an individual to default if they are in a mobile money treatment group, but come bearing cash to the meeting and do not have a balance in their BUBL account. How often this occurs will inform the Treatment-on-the-Treated estimation strategy proposed later in the PAP.

\subsection{Sub-Intervention}

As part of the baseline survey, borrowers were individually randomized into one of four different information treatments or a control group which did not receive a treatment.

These included:

- Control: No treatment

- Information Treatment: Respondents received an information treatment focused either on buying airtime using a mobile money account or on checking their balance using a mobile money account

- Security Treatment: Respondents received an information treatment focused either on reversing erroneous mobile money charges (i.e., charges sent to the wrong person) or changing the PIN on their existing mobile money account

In practice, trainings ranged from two to five minutes in length, and were given by the surveyor who conducted the respondent's baseline survey. Respondents were also allowed to opt out of or decline the information training, which was more common for the information treatments than the security treatments.

These treatments were used to predict mobile money-based take up (non-incentivized) offered in a hypothetical form as part of the survey. This data was available to researchers prior to specification of the PAP, so we do not include specifics looking at analysis within the baseline survey. Other uses of the sub-intervention will be detailed below.

\subsection{Treatment Assignment}


Treatment assignment was conducted on November 17, 2021. Randomization was group-level, stratified across the nine BUBL branches and satellites included in the study.

\section{Data Collection}

\subsection{Baseline Data}

The baseline survey for the study was conducted in September and October 2021. Surveying was done face-to-face, with surveyors meeting individually with borrowers.

The baseline survey covered basic demographics, including household composition and intra-household decision making processes, credit usage, business revenue, household income, group cohesion and experience, trust in financial institutions, education and literacy, mobile money knowledge and usage, a mobile money balance check activity, an incentivized risk aversion activity, an incentivized mobile money preferences assessment, the costs and benefits of mobile money repayment for a loan, the brief randomized information treatment described above, a non-incentivized (practice) choice between a hypothetical mobile money system and cash repayment for their BRAC loan, and a detailed module on intimate partner financial relationships and intimate partner violence.

In total, 2,100 women were surveyed for the baseline survey, roughly $20 \%$ of the sample as a whole. The baseline survey sample was selected from a list of borrowers at the nine branches provided in August 2021, and was a random sample stratified at the group level, such that every individual had the same odds of selection and each group was represented in the baseline survey. The only exclusions were very small groups - groups with one, two or three members who were not included in the sample.

\subsection{Mobile Money Choice Exercise}

From November 1 through November 15, surveyors accompanied credit officers to group meetings. All respondents at all groups in the microfinance branches were asked to make a choice between mobile money-based repayment and cash-based repayment for their loan, after a brief presentation on the specific mobile money payment system that BUBL would be utilizing.

Repayment modality choices were checked off on papers; a copy was given to the borrowers, and another copy was kept by the enumerators, who later entered the votes using QR codes. All respondents were given the opportunity to choose prior to randomization. Credit officers were told in advance to mobilize borrowers for the repayment choice, and to let borrowers know that not choosing would mean enrolling in mobile money-based repayment.

\subsection{Administrative Data}


As part of baseline activities, we are undertaking the digitalization of borrower household financial status surveys, conducted as part of BUBL's routine operations each time a borrower takes out a new loan.

Over the course of the study, BUBL will provide regular updates on the transactions made by each borrower. BUBL has also communicated their intent to provide group meeting attendance data for each borrower.

\subsection{Endline Data}

An endline survey is planned for July 2022, with the final date based on the rollout and collaboration with BUBL.

\section{Outcomes}

We differentiate between outcomes that will be drawn from the administrative data for all borrowers, and outcomes that will be drawn from the survey data for a subsample of borrowers. Note that where possible, we pre-specify the direction of tests; in practice, however, we intend to conduct two-sample tests for all outcome families.

I have broken down outcomes into families. For each outcome measure, we report the measures will be used to measure this, either independently or combined into an index as specified below.

\subsection{Administrative Data}

BUBL will provide administrative data on loan repayment throughout the course of the survey and has communicated their intent to provide group meeting attendance data as well.

\section{Outcome Family 1.1: Mobile Money Take Up}

Hypothesis 1.1: Treatment with mobile money repayment increases the likelihood and share of payments made using mobile money.

Measures: Whether a borrower is enabled in the BUBL system to transact between their mobile money wallet and BUBL bank account, whether a borrower ever makes a payment using mobile money, how many payments are made using mobile money out of the total number of payments during the study period.

\section{Outcome Family 1.2: Loan Performance}

Hypothesis 1.2: Treatment with mobile money repayment affects a borrower's loan performance.

Measures: Whether any payment is received late or made partially over the course of the study, the number of partial or late payments over the course of the study relative to the total number of payments due, average days in arrears as of endline, whether any loan is rated as anything 
other than a normal risk class by BUBL at endline, whether any account status is anything other than active at endline, whether any account is rated as bad debt at endline.

\section{Outcome Family 1.3: Loan Uptake}

Hypothesis 1.3: Treatment with mobile money repayment affects whether a borrower continues with BUBL as a client.

Measures: Whether the borrower still has an active loan as of the endline.

\section{Outcome Family 1.4: Group Attendance}

Hypothesis 1.4: Treatment with mobile money repayment decreases meeting attendance. Measure: Fraction of meetings attended by a borrower of the course of the study.

\section{Outcome Family 1.5: Account Balance}

Hypothesis 1.5: Treatment with mobile money repayment increases account balance. Measure: Account balance at endline, flow into bank account in the last month of the study, number of transactions made to bank account during last month of study.

\section{Outcome Family 1.6: Mobile Money Continuation}

Hypothesis 1.6: Treatment with mobile money repayment affects whether a borrower continues to want to repay via mobile money post-endline.

Measure: Whether the borrower would choose to continue to repay via mobile money after the end of the official study period. ${ }^{1}$

\section{Outcome Family 1.7: Group Membership Rates}

Hypothesis 1.7: Treatment with mobile money repayment affects group membership rates. Measure: Proportion of new borrowers who join a microfinance group, proportion of existing borrowers who exit a microfinance group.

\subsection{Survey Data}

\section{Outcome Family 2.1: Group Cohesion and Experience}

Hypothesis 2.1: Treatment with mobile money repayment affects microfinance group cohesion and borrower's experience of the group meeting.

Measures: Missed group meetings in the last three months, preference between attending group meeting and most-desired alternative use of time, frequency of repayment on behalf of other group members, length of group meeting time, time spent talking about business related matters, time spent talking about non-business related matters, active participation in conversation at meetings, whether meeting is considered useful for repayment, whether meeting is considered useful for other purposes, number of group members connecting with respondent outside group meeting in last two weeks, number of conversations about business matters in the last two weeks, number of conversations about personal matters in the last two weeks

\footnotetext{
${ }^{1}$ We hope to measure this outcome using administrative data; however, it is possible that we will instead need to use survey data.
} 


\section{Outcome Family 2.2: Mobile Money Knowledge and Usage}

Hypothesis 2.2: Treatment with mobile money repayment affects mobile money knowledge and use.

Measures: Frequency of mobile money use, knowledge of common mobile money transactions, frequency of usage in the past thirty days of mobile money for common tasks, whether the respondent reports using mobile money by themselves as opposed to with assistance or via an agent, whether respondent accurately answered questions regarding withdrawal fees, whether respondent could complete a mobile money balance check, whether respondent preferred cash or mobile money in an incentivized choice task (see Appendix for details on mobile money frequency of use and knowledge).

\section{Outcome Family 2.3: Mobile Money Perceptions}

Hypothesis 2.3: Treatment with mobile money repayment affects perceptions of mobile money. Measures: Respondent agrees with positive statements on mobile money use, respondent disagrees with negative statements about mobile money use, respondent rates positively the benefits of mobile money, respondent rates reasons around using mobile money as important (see Appendix for details)

\section{Outcome Family 2.4: Business Performance}

Hypothesis 2.4: Treatment with mobile money affects business performance. Measures: Share of loan spent on own business, has a current business, total revenue of business in last six months, total revenue of business in last thirty days, business revenue has increased over the last thirty days, satisfied or very satisfied with business revenue

\section{Outcome Family 2.5: Household Income and Consumption}

Hypothesis 2.5: Treatment with mobile money affects household income and consumption. Measures: Total income per month of the respondent, total income per month of the household, money spent on food in last seven days, money spent on fuel in last thirty days

\section{Outcome Family 2.6: Female Empowerment}

Hypothesis 2.6: Treatment with mobile money affects female empowerment.

Outcomes: Rates self as most important person in deciding whether to take loan, rates self as most important person in making decision to borrower, rates self as most important person in deciding on usage of loan, rates self as primary decision maker in daily household expenses, rates self as primary decision maker in case of emergencies

\section{Outcome Family 2.7: Intimate Partner Financial Control and Violence}

Hypothesis 2.7: Treatment with mobile money affects intimate partner financial control and violence.

Outcomes: Measurements of intimate partner financial control and intimate partner violence (see Appendix for details)

\section{Analysis}




\subsection{Estimation Methodology}

McKenzie (2012) showed that in the case of a single baseline and follow-up with an autocorrelation less than 0.5 , power is highest when regressing an outcome measure at endline on baseline covariates, the treatment indicator and the baseline value of the outcome measure. There are large power gains from using ANCOVA rather than a difference-in-difference specification. The study will therefore be analyzed using an OLS regression of the form:

$$
Y_{i 1}=\beta_{0}+\beta_{1} T_{j}^{1}+\beta_{2} T_{j}^{2}+Y_{i 0}+\lambda_{j}+X_{i 0}+\varepsilon_{i j}
$$

where $Y_{i 1}$ is the outcome of interest for individual $i$ in group $j$ measured at endline, $T_{j}^{1}$ the mobile money-based repayment treatment dummy, $T_{j}^{2}$ the individual choice repayment treatment dummies, $\lambda_{i}$ a set of randomization strata dummies (Bruhn and McKenzie, 2009), $Y_{i 0}$ is the baseline value of the outcome (if measured at baseline, otherwise excluded) and $\varepsilon_{i}$ random error for individual $i$. In some specifications, a set of covariates $X_{i 0}$ selected using Least Absolute Shrinkage and Selection Operator (LASSO) from the pre-study administrative data or baseline survey will be included to improve precision (Bloniarz et. al., 2016). Standard errors will be clustered at the microfinance group level, which is the unit of randomization.

OLS estimations of the above regression will return the unbiased estimate of Intent to Treat (ITT) estimates of $\beta_{1}$ and $\beta_{2}$. To estimate the local average treatment effect (or treatment on the treated), assuming no spillovers, we will estimate the above equation where assignment to treatment is replaced by actual take-up instrumented by assignment, giving the two-stage least squares estimator.

For selected outcome families, we will estimate a group-level regression of the form:

$$
Y_{j 1}=\beta_{0}+\beta_{1} T_{j}^{1}+\beta_{2} T_{j}^{2}+Y_{i 0}+\lambda_{j}+X_{j 0}+\varepsilon_{j}
$$

where variables are defined as in equation (1), at the level of the group $j$ rather than the individual $i$.

\subsection{Hypothesis Testing}

For each outcome, we will test the following hypotheses:

1. Whether being assigned to repay with mobile money has an effect $\left(H_{0}: \beta_{1}=0\right)$

2. Whether being assigned to have one's individual choice on mobile money has any effect $\left(H_{0}: \beta_{2}=0\right)$

3. Whether these differ $\left(H_{0}: \beta_{1}=\beta_{2}\right)$ 


\subsection{Multiple Test Correction}

As this study examines effects of the treatments on various outcome measures, we will adjust the $p$-values of the coefficients of interest for multiple inference by calculating sharpened $q$-values that control for the false discovery rate (FDR) within each outcome family. Rather than pre-specifying a single q, we report the minimum q-value at which each hypothesis is rejected, following Anderson (2008) and Benjamini et al. (2006), calculated across the number of outcomes per interaction term.

\subsection{Index Construction}

For most outcome families, we will group several related variables into index variables, following Anderson (2008). We will construct the indices in three steps. First, we re-code all outcomes so that higher values correspond to treatment effects in the same direction ("better" outcomes). Second, we will standardize individual outcomes using the baseline mean and standard deviation for that outcome. Third, we will calculate the average of the standardized constituent outcomes. Where an outcome value is missing for a respondent, we will omit this outcome from the index construction.

\subsection{Heterogeneous Effects}

We also test whether the impact of the treatment varies by characteristics of the borrowers as measured at baseline by augmenting equation (1) with the baseline measure of interest and an interaction between the treatment indicators and the baseline measure of interest.

Specifically, we will examine heterogeneous effects by the following measures for the whole sample:

- Stratification variables (i.e., which branch a respondent belongs to)

- Pre-baseline index of loan performance ${ }^{2}$

- An indicator variable equal to one if the respondent chose to repay with mobile money rather than cash

- An index of respondent socio-economic status from loan intake forms (see Appendix)

- Amount that respondent self-reported being able to pay weekly in loan intake form

- Amount that respondent self-reported in weekly net sales in loan intake form

- Age of respondent business in years self-reported in loan intake form

For the survey sample only, we will look at heterogeneity across the following measures:

- An indicator variable equal to one if the respondent was married

- An indicator variable equal to one if the respondent reported disliking group meetings at baseline

- Baseline index of mobile money knowledge and use

- Baseline index of mobile money perceptions

\footnotetext{
${ }^{2}$ Measured over the six months prior to the beginning of the study
} 
- Baseline index of intimate partner financial control and violence

- Baseline index of female empowerment

- Baseline index of group cohesion and experience

- Baseline financial literacy activity score

- Baseline risk aversion

For heterogeneous effects, we will adjust the $p$-values of the coefficients of interest for multiple inference by calculating sharpened q-values that control for the false discovery rate (FDR) within each outcome family. Rather than pre-specifying a single $q$, we report the minimum q-value at which each hypothesis is rejected, following Anderson (2008) and Benjamini et al. (2006), calculated across the number of outcomes per interaction term.

\subsection{Spillover Estimation}

The payment modality choice exercise conducted prior to randomization will yield natural, known variation in the proportion of other individuals in one's group randomized into mobile money in the Individual Choice Treatment. In practice, it will also be known for other treatment arms how many individuals would have chosen cash vs. mobile money if able to, allowing us to control for the potential endogeneity of group effects inherent in the choice.

Potential spillovers will therefore by analyzed using an OLS regression of the following form:

$$
Y_{i 1}=\alpha_{0}+\alpha_{1} \eta_{j}+\alpha_{2} T_{i}^{1}+\alpha_{3} T_{i}^{1} \times \eta_{j}+\alpha_{4} T_{i}^{2}+\alpha_{5} T_{i}^{2} \times \eta_{j}+X_{i}+Y_{i 0}+\varepsilon_{i j}
$$

where $Y_{i j 1}$ is the outcome of interest for individual $i$ in group measured at endline, $\eta_{j}$ is the fraction of one's group $j$ who chose mobile money-based repayment prior to randomization, $T_{i}^{1}$ the mobile money-based repayment treatment dummy, $T_{i}^{2}$ the individual choice repayment treatment dummies, $X_{i}$ a set of randomization strata dummies (Bruhn and McKenzie, 2009), $Y_{i 0}$ is the baseline value of the outcome (if measured at baseline, otherwise excluded) and $\varepsilon_{i}$ random error for individual $i$. Standard errors will be clustered the microfinance group level, which is the unit of randomization.

In this specification, $\alpha_{1}$ will estimate the correlation between share of mobile money repayment uptake and the outcome of interest among the control group, $\alpha_{3}$ will estimate the difference in treatment effect as the share of uptake increases within the groups where all members are asked to repay with mobile money, and $\alpha_{5}$ will estimate the difference in treatment effect as the share of uptake increases within the groups where individual choices are implemented. 
Under perfect compliance, we would expect to detect no spillovers in any but the individual choice repayment arm; under imperfect compliance, we might expect to detect spillovers as well in the mobile money-based repayment arm.

We plan to estimate this regression for outcome family 1 , outcome family 2 , outcome family 3 , outcome family 4 , outcome family 5 , outcome family 6 , outcome family 7 and outcome family 8 . For these specifications, we will adjust the $p$-values of the coefficients of interest for multiple inference by calculating sharpened q-values that control for the false discovery rate (FDR) within each outcome family. Rather than pre-specifying a single $q$, we report the minimum q-value at which each hypothesis is rejected, following Anderson (2008) and Benjamini et al. (2006), calculated across the number of outcomes per interaction term.

\section{Robustness and Attrition}

\subsection{Robustness}

I will perform the following robustness checks:

- If any covariate was found to be unbalanced at baseline, I will compare equation (1) with and without that covariate for my survey sample.

- I will construct $p$-values using a permutation test based on a randomization inference approach.

\subsection{Adjusting for missing data in follow-up surveys}

I will present main results without adjustment for attrition (e.g., households not surveyed in the follow-up) or non-response (e.g., individual questions not answered in the follow-up).

For any individual outcome missing for more than $15 \%$ of the sample, I will compare the fraction of missing data by assigned treatment status. I do this by estimating equation (1) using an indicator for attrition as an outcome (and omitting $Y_{i 0}$ and $X_{i 0}$ ) and testing if the coefficients on the treatment dummies equal zero. This will allow me to assess differential non-response across treatment and control.

\section{Appendix: Outcome Measures}

In this appendix, we provide additional information on some outcome measures.

\section{Outcome Family 2.2: Mobile Money Knowledge and Usage}

Hypothesis 2.2: Treatment with mobile money repayment affects mobile money knowledge and use.

Knows how to:

- Airtime recharge

- Deposit cash 
- Withdraw cash

- Send money

- Receive money

- Bill payment

- Push money to a bank account

- Pull money from your bank account

- Contact customer service

- Correct incorrectly sent money

- Change PIN number

Frequency of usage in the past thirty days of mobile money for:

- Airtime recharge

- Deposit cash

- Withdraw cash

- Send money

- Receive money

- Bill payment

- Push money to a bank account

- Pull money from your bank account

\section{Outcome Family 2.3: Mobile Money Perceptions}

Hypothesis 2.3: Treatment with mobile money repayment affects perceptions of mobile money. Respondent agrees with positive statements on mobile money use:

- Mobile money is easy to use

- Mobile money is safer than keeping cash

- Mobile money saves time

- Mobile money reduces travel costs

- Mobile money is cheaper than the other ways I send or receive money from friends and family

Respondent disagrees with negative statements about mobile money use:

- No one among my friends or family uses mobile money

- I have no need for mobile money

- I do not trust that my money is safe with mobile money

- Mobile money is too expensive

Respondent rates positively the benefits of mobile money:

- Cost saving

- Time saving

- $24 \mathrm{~h}$ access

- Physical security

Respondent rates reasons around using mobile money as important:

- Lower transaction costs

- Security from fraud

- Physical security

- Safe transaction with feedback on transfer

- Wide acceptance of mobile money

- More locations I can cash-out my money 


\section{Outcome Family 2.7: Intimate Partner Financial Control and Violence}

Hypothesis 2.7: Treatment with mobile money affects intimate partner financial control and violence.

Intimate partner financial control: woman indicates that in the last six months, their partner sometimes or often would:

- Expect that you ask permission to purchase large items?

- Expect that you ask permission to purchase small items?

- Take your earnings or savings from you against your will?

- Refuse to give you money for household expenses, even when he had money for other things?

- Require that you give up or refuse a job for money outside the home because your partner does not want you to work?

- Make important financial decisions without talking to you about them?

- Decide how you could spend money?

- Demand to know how you spent money?

- Hide money from you?

- Spent money you needed for rent, food or clothes on things for him?

- Threaten to not give you money or take it away from you?

- Given you little money or reduced your spending when he is angry?

- Stopped giving you money for food?

Intimate partner violence: respondent indicates that in the last six months, their sometimes or often would:

- Push you, shake you, or throw something at you?

- Slap you?

- Twist your arm or pull your hair?

- Punch you with his first or with something that could hurt you?

- Kick you, drag you, or beat you up?

- Try to choke you or burn you on purpose?

- Threaten or attack you with a knife, gun, or other weapon?

- Physically force you to have sexual intercourse with him when you did not want to?

- Physically force you to perform any other sexual acts you did not want to?

- Force you with threats or in any other way to perform sexual acts you did not want to?

\section{Respondent Socio-Economic Status Index}

- Number of household members

- Whether walls were made with a material other than mud

- Whether roof was made with a material other than thatch or tins

- Whether charcoal or gas is used for cooking

- Whether the household has a covered toilet

- Whether the household owns two or more phones

- Whether the household owns a radio

- Whether every member of the household has at least one pair of shoes 


\section{References}

Anderson, M. L. (2008). Multiple Inference and Gender Differences in the Effects of Early Intervention: A Reevaluation of the Abecedarian, Perry Preschool, and Early Training Projects. Journal of the American Statistical Association, 103(484):1481-1495.

Benjamini, Y., Krieger, A. M., and Yekutieli, D. (2006). Adaptive linear step-up procedures that control the false discovery rate. Biometrika, 93(3):491-507.

Bruhn, M. and McKenzie, D. (2009). In Pursuit of Balance: Randomization in Practice in Development Field Experiments. American Economic Journal: Applied Economics, 1(4):200-232.

Bloniarz, A., Liu, H., Zhang, C., Sekhon, J. S., Yu, B. (2016). Lasso adjustments of treatment effect estimates in randomized experiments. Procedures of the National Academy of Sciences, USA, 113(27):7838-7390.

McKenzie, D. (2012). Beyond baseline and follow-up: the case for more $T$ in experiments. Journal of Development Economics, 99(2):210-221. 